\title{
Numerical Methods for Solving Fredholm Integral Equations of Second Kind
}

\author{
S. Saha Ray and P. K. Sahu \\ Department of Mathematics, National Institute of Technology, Rourkela 769008, India \\ Correspondence should be addressed to S. Saha Ray; santanusaharay@yahoo.com
}

Received 3 September 2013; Accepted 3 October 2013

Academic Editor: Rasajit Bera

Copyright (c) 2013 S. S. Ray and P. K. Sahu. This is an open access article distributed under the Creative Commons Attribution License, which permits unrestricted use, distribution, and reproduction in any medium, provided the original work is properly cited.

Integral equation has been one of the essential tools for various areas of applied mathematics. In this paper, we review different numerical methods for solving both linear and nonlinear Fredholm integral equations of second kind. The goal is to categorize the selected methods and assess their accuracy and efficiency. We discuss challenges faced by researchers in this field, and we emphasize the importance of interdisciplinary effort for advancing the study on numerical methods for solving integral equations.

\section{Introduction}

Integral equations occur naturally in many fields of science and engineering [1]. A computational approach to solve integral equation is an essential work in scientific research.

Integral equation is encountered in a variety of applications in many fields including continuum mechanics, potential theory, geophysics, electricity and magnetism, kinetic theory of gases, hereditary phenomena in physics and biology, renewal theory, quantum mechanics, radiation, optimization, optimal control systems, communication theory, mathematical economics, population genetics, queuing theory, medicine, mathematical problems of radiative equilibrium, the particle transport problems of astrophysics and reactor theory, acoustics, fluid mechanics, steady state heat conduction, fracture mechanics, and radiative heat transfer problems. Fredholm integral equation is one of the most important integral equations.

Integral equations can be viewed as equations which are results of transformation of points in a given vector spaces of integrable functions by the use of certain specific integral operators to points in the same space. If, in particular, one is concerned with function spaces spanned by polynomials for which the kernel of the corresponding transforming integral operator is separable being comprised of polynomial functions only, then several approximate methods of solution of integral equations can be developed.

A computational approach to solving integral equation is an essential work in scientific research. Some methods for solving second kind Fredholm integral equation are available in the open literature. The $B$-spline wavelet method, the method of moments based on $B$-spline wavelets by Maleknejad and Sahlan [2], and variational iteration method (VIM) by He [3-5] have been applied to solve second kind Fredholm linear integral equations. The learned researchers Maleknejad et al. proposed some numerical methods for solving linear Fredholm integral equations system of second kind using Rationalized Haar functions method, Block-Pulse functions, and Taylor series expansion method [6-8]. Haar wavelet method with operational matrices of integration [9] has been applied to solve system of linear Fredholm integral equations of second kind. Quadrature method [10], B-spline wavelet method [11], wavelet Galerkin method [12], and also VIM [13] can be applied to solve nonlinear Fredholm integral equation of second kind. Some iterative methods like Homotopy perturbation method (HPM) [14-16] and Adomian decomposition method (ADM) [16-18] have been applied to solve nonlinear Fredholm integral equation of second kind. 


\section{Fredholm Integral Equation}

The general form of linear Fredholm integral equation is defined as follows:

$$
g(x) y(x)=f(x)+\lambda \int_{a}^{b} K(x, t) y(t) d t
$$

where $a$ and $b$ are both constants. $f(x), g(x)$, and $K(x, t)$ are known functions while $y(x)$ is unknown function. $\lambda$ (nonzero parameter) is called eigenvalue of the integral equation. The function $K(x, t)$ is known as kernel of the integral equation.

2.1. Fredholm Integral Equation of First Kind. The linear integral equation is of form (by setting $g(x)=0$ in (1))

$$
f(x)+\lambda \int_{a}^{b} K(x, t) y(t) d t=0 .
$$

Equation (2) is known as Fredholm integral equation of first kind.

2.2. Fredholm Integral Equation of Second Kind. The linear integral equation is of form (by setting $g(x)=1$ in (1))

$$
y(x)=f(x)+\int_{a}^{b} K(x, t) y(t) d t
$$

Equation (3) is known as Fredholm integral equation of second kind.

2.3. System of Linear Fredholm Integral Equations. The general form of system of linear Fredholm integral equations of second kind is defined as follows:

$$
\begin{array}{r}
\sum_{j=1}^{n} g_{i, j} y_{j}(x)=f_{i}(x)+\sum_{j=1}^{n} \int_{a}^{b} K_{i, j}(x, t) y_{j}(t) d t \\
i=1,2, \ldots, n
\end{array}
$$

where $f_{i}(x)$ and $K_{i, j}(x, t)$ are known functions and $y_{j}(x)$ are the unknown functions for $i, j=1,2, \ldots, n$.

2.4. Nonlinear Fredholm-Hammerstein Integral Equation of Second Kind. Nonlinear Fredholm-Hammerstein integral equation of second kind is defined as follows:

$$
y(x)=f(x)+\int_{a}^{b} K(x, t) F(y(t)) d t,
$$

where $K(x, t)$ is the kernel of the integral equation, $f(x)$ and $K(x, t)$ are known functions, and $y(x)$ is the unknown function that is to be determined.
2.5. System of Nonlinear Fredholm Integral Equations. System of nonlinear Fredholm integral equations of second kind is defined as follows:

$$
\begin{array}{r}
\sum_{j=1}^{n} g_{i, j} y_{j}(x)=f_{i}(x)+\sum_{j=1}^{n} \int_{a}^{b} K_{i, j}(x, t) F_{i, j}\left(t, y_{j}(t)\right) d t \\
i=1,2, \ldots, n,
\end{array}
$$

where $f_{i}(x)$ and $K_{i, j}(x, t)$ are known functions and $y_{j}(x)$ are the unknown functions for $i, j=1,2, \ldots, n$.

\section{Numerical Methods for Linear Fredholm Integral Equation of Second Kind}

Consider the following Fredholm integral equation of second kind defined in (3)

$$
y(x)=f(x)+\int_{a}^{b} K(x, t) y(t) d t, \quad a \leq x \leq b,
$$

where $K(x, t)$ and $g(x)$ are known functions and $y(x)$ is unknown function to be determined.

\subsection{B-Spline Wavelet Method}

3.1.1. B-Spline Scaling and Wavelet Functions on the Interval $[0,1]$. Semiorthogonal wavelets using $B$-spline are specially constructed for the bounded interval and this wavelet can be represented in a closed form. This provides a compact support. Semiorthogonal wavelets form the basis in the space $L^{2}(R)$.

Using this basis, an arbitrary function in $L^{2}(R)$ can be expressed as the wavelet series. For the finite interval $[0,1]$, the wavelet series cannot be completely presented by using this basis. This is because supports of some basis are truncated at the left or right end points of the interval. Hence, a special basis has to be introduced into the wavelet expansion on the finite interval. These functions are referred to as the boundary scaling functions and boundary wavelet functions.

Let $m$ and $n$ be two positive integers and let

$$
\begin{aligned}
a & =x_{-m+1}=\cdots=x_{0}<x_{1} \\
& <\cdots<x_{n}=x_{n+1} \\
& =\cdots=x_{n+m-1}=b
\end{aligned}
$$

be an equally spaced knots sequence. The functions

$$
\begin{gathered}
B_{m, j, X}(x)=\frac{x-x_{j}}{x_{j+m-1}-x_{j}} B_{m-1, j, X}(x) \\
+\frac{x_{j+m}-x}{x_{j+m}-x_{j+1}} B_{m-1, j+1, X}(x), \\
j=-m+1, \ldots, n-1, \\
B_{1, j, X}(x)= \begin{cases}1, & x \in\left[x_{j}, x_{j+1}\right), \\
0, & \text { otherwise, }\end{cases}
\end{gathered}
$$


are called cardinal $B$-spline functions of order $m \geq 2$ for the knot sequence $X=\left\{x_{i}\right\}_{i=-m+1}^{n+m-1}$ and $\operatorname{Supp} B_{m, j, X}(x)=$ $\left[x_{j}, x_{j+m}\right] \cap[a, b]$.

By considering the interval $[a, b]=[0,1]$, at any level $j \in$ $\mathrm{Z}^{+}$, the discretization step is $2^{-j}$, and this generates $n=2^{j}$ number of segments in $[0,1]$ with knot sequence

$$
X^{(j)}=\left\{\begin{array}{l}
x_{-m+1}^{(j)}=\cdots=x_{0}^{(j)}=0 \\
x_{k}^{(j)}=\frac{k}{2^{j}}, \quad k=1, \ldots, n-1, \\
x_{n}^{(j)}=\cdots=x_{n+m-1}^{(j)}=1 .
\end{array}\right.
$$

Let $j_{0}$ be the level for which $2^{j_{0}} \geq 2 m-1$; for each level, $j \geq j_{0}$, the scaling functions of order $m$ can be defined as follows in [2]:

$$
\begin{aligned}
\varphi_{m, j, i} & (x) \\
= & \begin{cases}B_{m, j_{0}, i}\left(2^{j-j_{0}} x\right) & i=-m+1, \ldots,-1, \\
B_{m, j_{0}, 2^{j}-m-i}\left(1-2^{j-j_{0}} x\right) & i=2^{j}-m+1, \ldots, 2^{j}-1, \\
B_{m, j_{0}, 0}\left(2^{j-j_{0}} x-2^{-j_{0}} i\right) & i=0, \ldots, 2^{j}-m .\end{cases}
\end{aligned}
$$

And the two scale relations for the $m$-order semiorthogonal compactly supported $B$-wavelet functions are defined as follows:

$$
\begin{gathered}
\psi_{m, j, i-m}=\sum_{k=i}^{2 i+2 m-2} q_{i, k} B_{m, j, k-m}, \quad i=1, \ldots, m-1, \\
\psi_{m, j, i-m}=\sum_{k=2 i-m}^{2 i+2 m-2} q_{i, k} B_{m, j, k-m}, \quad i=m, \ldots, n-m+1, \\
\psi_{m, j, i-m}=\sum_{k=2 i-m}^{n+i+m-1} q_{i, k} B_{m, j, k-m}, \quad i=n-m+2, \ldots, n,
\end{gathered}
$$

where $q_{i, k}=q_{k-2 i}$.

Hence, there are $2(m-1)$ boundary wavelets and $(n-$ $2 m+2)$ inner wavelets in the bounded interval $[a, b]$. Finally, by considering the level $j$ with $j \geq j_{0}$, the $B$-wavelet functions in $[0,1]$ can be expressed as follows:

$$
\begin{aligned}
& \psi_{m, j, i}(x) \\
& = \begin{cases}\psi_{m, j_{0}, i}\left(2^{j-j_{0}} x\right) & i=-m+1, \ldots,-1, \\
\psi_{m, 2^{j}-2 m+1-i, i}\left(1-2^{j-j_{0}} x\right) & i=2^{j}-2 m+2, \ldots, 2^{j}-m, \\
\psi_{m, j_{0}, 0}\left(2^{j-j_{0}} x-2^{-j_{0}} i\right) & i=0, \ldots, 2^{j}-2 m+1 .\end{cases}
\end{aligned}
$$

The scaling functions $\varphi_{m, j, i}(x)$ occupy $m$ segments and the wavelet functions $\psi_{m, j, i}(x)$ occupy $2 m-1$ segments.

When the semiorthogonal wavelets are constructed from $B$-spline of order $m$, the lowest octave level $j=j_{0}$ is determined in $[19,20]$ by

$$
2^{j_{0}} \geq 2 m-1
$$

so as to have a minimum of one complete wavelet on the interval $[0,1]$.
3.1.2. Function Approximation. A function $f(x)$ defined over $[0,1]$ may be approximated by $B$-spline wavelets as $[21,22]$

$$
\begin{aligned}
f(x)= & \sum_{k=1-m}^{2^{j_{0}}-1} c_{j_{0}, k} \varphi_{j_{0}, k}(x) \\
& +\sum_{j=j_{0}}^{\infty} \sum_{k=1-m}^{2^{j}-m} d_{j, k} \psi_{j, k}(x) .
\end{aligned}
$$

If the infinite series in (15) is truncated at $M$, then (15) can be written as [2]

$$
\begin{aligned}
f(x) \cong & \sum_{k=1-m}^{2^{j_{0}}-1} c_{j_{0}, k} \varphi_{j_{0}, k}(x) \\
& +\sum_{j=j_{0}}^{M} \sum_{k=1-m}^{2^{j}-m} d_{j, k} \psi_{j, k}(x),
\end{aligned}
$$

where $\varphi_{2, k}$ and $\psi_{j, k}$ are scaling and wavelets functions, respectively, and $C$ and $\Psi$ are $\left(2^{M+1}+m-1\right) \times 1$ vectors given by

$$
\begin{gathered}
C=\left[c_{j_{0}, 1-m}, \ldots, c_{j_{0}, 2^{j_{0}-1}}, d_{j_{0}, 1-m}, \ldots,\right. \\
\left.d_{j_{0}, 2^{j_{0}}-m}, \ldots, d_{M, 1-m}, \ldots, d_{M, 2^{M}-m}\right]^{T}, \\
\Psi=\left[\varphi_{j_{0}, 1-m}, \ldots, \varphi_{j_{0}, 2^{j_{0}-1}}, \psi_{j_{0}, 1-m}, \ldots,\right. \\
\left.\psi_{j_{0}, 2^{j_{0}}-m}, \ldots, \psi_{M, 1-m}, \ldots, \psi_{M, 2^{M}-m}\right]^{T},
\end{gathered}
$$

with

$$
\begin{gathered}
c_{j_{0}, k}=\int_{0}^{1} f(x) \widetilde{\varphi}_{j_{0}, k}(x) d x, \quad k=1-m, \ldots, 2^{j_{0}}-1, \\
d_{j, k}=\int_{0}^{1} f(x) \widetilde{\psi}_{j, k}(x) d x, \\
j=j_{0}, \ldots, M, \quad k=1-m, \ldots, 2^{M}-m,
\end{gathered}
$$

where $\widetilde{\varphi}_{j_{0}, k}(x)$ and $\widetilde{\psi}_{j, k}(x)$ are dual functions of $\varphi_{j_{0}, k}$ and $\psi_{j, k}$, respectively. These can be obtained by linear combinations of $\varphi_{j_{0}, k}, k=1-m, \ldots, 2^{j_{0}}-1$, and $\psi_{j, k}, j=j_{0}, \ldots, M, k=$ $1-m, \ldots, 2^{M}-m$, as follows. Let

$$
\Phi=\left[\varphi_{j_{0}, 1-m}, \ldots, \varphi_{j_{0}, 2^{j_{0}}-1}\right]^{T},
$$

$$
\bar{\Psi}=\left[\psi_{j_{0}, 1-m}, \ldots, \psi_{j_{0}, 2^{j_{0}}-m}, \ldots, \psi_{M, 1-m}, \ldots, \psi_{M, 2^{M}-m}\right]^{T} \text {. }
$$

Using (11), (20), (12)-(13), and (21), we get

$$
\begin{aligned}
& \int_{0}^{1} \Phi \Phi^{T} d x=P_{1} \\
& \int_{0}^{1} \bar{\Psi} \bar{\Psi}^{T} d x=P_{2} .
\end{aligned}
$$


Suppose that $\widetilde{\Phi}$ and $\widetilde{\Psi}$ are the dual functions of $\Phi$ and $\bar{\Psi}$, respectively; then

$$
\begin{gathered}
\int_{0}^{1} \widetilde{\Phi} \Phi^{T} d x=I_{1}, \\
\int_{0}^{1} \widetilde{\Psi} \Psi^{T} d x=I_{2}, \\
\widetilde{\Phi}=P_{1}^{-1} \Phi, \\
\widetilde{\Psi}=P_{2}^{-1} \bar{\Psi} .
\end{gathered}
$$

3.1.3. Application of B-Spline Wavelet Method. In this section, linear Fredholm integral equation of the second kind of form (7) has been solved by using $B$-spline wavelets. For this, we use (16) to approximate $y(x)$ as

$$
y(x)=C^{T} \Psi(x),
$$

where $\Psi(x)$ is defined in (18) and $C$ is $\left(2^{M+1}+m-1\right) \times 1$ unknown vector defined similarly as in (17). We also expand $f(x)$ and $K(x, t)$ by $B$-spline dual wavelets $\widetilde{\Psi}$ defined in (24) as

$$
\begin{gathered}
f(x)=C_{1}{ }^{T} \widetilde{\Psi}(x), \\
K(x, t)=\widetilde{\Psi}^{T}(t) \Theta \widetilde{\Psi}(x),
\end{gathered}
$$

where

$$
\Theta_{i, j}=\int_{0}^{1}\left[\int_{0}^{1} K(x, t) \Psi_{i}(t) d t\right] \Psi_{j}(x) d x .
$$

From (26) and (25), we get

$$
\begin{aligned}
\int_{0}^{1} K(x, t) y(t) d t & =\int_{0}^{1} C^{T} \Psi(t) \widetilde{\Psi}^{T}(t) \Theta \widetilde{\Psi}(x) d t \\
& =C^{T} \Theta \widetilde{\Psi}(x)
\end{aligned}
$$

since

$$
\int_{0}^{1} \Psi(t) \widetilde{\Psi}^{T}(t) d t=I
$$

By applying (25)-(28) in (7) we have

$$
C^{T} \Psi(x)-C^{T} \Theta \widetilde{\Psi}(x)=C_{1}^{T} \widetilde{\Psi}(x) .
$$

By multiplying both sides of (30) with $\Psi^{T}(x)$ from the right and integrating both sides with respect to $x$ from 0 to 1 , we get

$$
C^{T} P-C^{T} \Theta=C_{1}^{T}
$$

since

$$
\int_{0}^{1} \widetilde{\Psi}(x) \Psi^{T}(x) d x=I,
$$

and $P$ is a $\left(2^{M+1}+m-1\right) \times\left(2^{M+1}+m-1\right)$ square matrix given by

$$
P=\int_{0}^{1} \Psi(x) \Psi^{T}(x) d x=\left(\begin{array}{cc}
P_{1} & 0 \\
0 & P_{2}
\end{array}\right)
$$

Consequently, from (31), we get $C^{T}=C_{1}^{T}(P-\Theta)^{-1}$. Hence, we can calculate the solution for $y(x)=C^{T} \Psi(x)$.

\subsection{Method of Moments}

3.2.1. Multiresolution Analysis (MRA) and Wavelets [2]. A set of subspaces $\left\{V_{j}\right\}_{j \in Z}$ is said to be MRA of $L^{2}(R)$ if it possesses the following properties:

$$
\begin{gathered}
V_{j} \subset V_{j+1}, \quad \forall j \in Z, \\
\bigcup_{j \in Z} V_{j} \text { is dense in } L^{2}(R), \\
\bigcap_{j \in Z} V_{j}=\phi, \\
f(x) \in V_{j} \Longleftrightarrow f(2 x) \in V_{j+1}, \quad \forall j \in Z,
\end{gathered}
$$

where $Z$ denotes the set of integers. Properties (34)-(36) state that $\left\{V_{j}\right\}_{j \in Z}$ is a nested sequence of subspaces that effectively covers $L^{2}(R)$. That is, every square integrable function can be approximated as closely as desired by a function that belongs to at least one of the subspaces $V_{j}$. A function $\varphi \in L^{2}(R)$ is called a scaling function if it generates the nested sequence of subspaces $V_{j}$ and satisfies the dilation equation; namely,

$$
\varphi(x)=\sum_{k} p_{k} \varphi(a x-k)
$$

with $p_{k} \in l^{2}$ and $a$ being any rational number.

For each scale $j$, since $V_{j} \subset V_{j+1}$, there exists a unique orthogonal complementary subspace $W_{j}$ of $V_{j}$ in $V_{j+1}$. This subspace $W_{j}$ is called wavelet subspace and is generated by $\psi_{j, k}=\psi\left(2^{j} x-k\right)$, where $\psi \in L^{2}$ is called the wavelet. From the above discussion, these results follow easily:

$$
\begin{array}{ll}
V_{j_{1}} \cap V_{j_{2}}=V_{j_{2}}, & j_{1}>j_{2}, \\
W_{j_{1}} \cap W_{j_{2}}=0, & j_{1} \neq j_{2}, \\
V_{j_{1}} \cap W_{j_{2}}=0, & j_{1} \leq j_{2} .
\end{array}
$$

Some of the important properties relevant to the present analysis are given below $[2,19]$.

(1) Vanishing Moment. A wavelet is said to have a vanishing moment of order $m$ if

$$
\int_{-\infty}^{\infty} x^{p} \psi(x) d x=0 ; \quad p=0, \ldots, m-1 .
$$


All wavelets must satisfy the previously mentioned condition for $p=0$.

(2) Semiorthogonality. The wavelets $\psi_{j, k}$ form a semiorthogonal basis if

$$
\left\langle\psi_{j, k}, \psi_{s, i}\right\rangle=0 ; \quad j \neq s ; \forall j, k, s, i \in \mathrm{Z} .
$$

3.2.2. Method of Moments for the Solution of Fredholm Integral Equation. In this section, we solve the integral equation of form (7) in interval $[0,1]$ by using linear $B$-spline wavelets [2]. The unknown function in (7) can be expanded in terms of the scaling and wavelet functions as follows:

$$
\begin{aligned}
y(x) \approx & \sum_{k=-1}^{2^{j_{0}}-1} c_{k} \varphi_{j_{0}, k}(x) \\
& +\sum_{j=j_{0}}^{M} \sum_{k=-1}^{2^{j}-2} d_{j, k} \psi_{j, k}(x) \\
= & C^{T} \Psi(x) .
\end{aligned}
$$

By substituting this expression into (7) and employing the Galerkin method, the following set of linear system of order $\left(2^{M}+1\right)$ is generated. The scaling and wavelet functions are used as testing and weighting functions:

$$
\left(\begin{array}{cc}
\langle\varphi, \varphi\rangle-\langle K \varphi, \varphi\rangle & \langle\psi, \varphi\rangle-\langle K \psi, \varphi\rangle \\
\langle\varphi, \psi\rangle-\langle K \varphi, \psi\rangle & \langle\psi, \psi\rangle-\langle K \psi, \psi\rangle
\end{array}\right)\left(\begin{array}{c}
C \\
D
\end{array}\right)=\left(\begin{array}{l}
F_{1} \\
F_{2}
\end{array}\right),
$$

where

$$
\begin{gathered}
C=\left[c_{-1}, c_{0}, \ldots, c_{3}\right]^{T}, \\
D=\left[d_{2,-1}, \ldots, d_{2,2}, d_{3,-1}, \ldots, d_{3,6}, \ldots,\right. \\
\left.d_{M,-1}, \ldots, d_{M, 2^{M}-2}\right]^{T}, \\
\langle\varphi, \varphi\rangle-\langle K \varphi, \varphi\rangle \\
=\left(\int_{0}^{1} \varphi_{j_{0}, r}(x) \varphi_{j_{0}, i}(x) d x\right. \\
\left.-\int_{0}^{1} \varphi_{j_{0}, r}(x) \int_{0}^{1} K(x, t) \varphi_{j_{0}, i}(t) d t d x\right)_{i, r} \\
\langle\psi, \varphi\rangle-\langle K \psi, \varphi\rangle \\
=\left(\int_{0}^{1} \varphi_{j_{0}, r}(x) \psi_{k, j}(x) d x\right. \\
\left.-\int_{0}^{1} \varphi_{j_{0}, r}(x) \int_{0}^{1} K(x, t) \psi_{k, j}(t) d t d x\right)_{r, k, j}, \\
\langle\varphi, \psi\rangle-\langle K \varphi, \psi\rangle \\
=\left(\int_{0}^{1} \psi_{s, l}(x) \varphi_{j_{0}, i}(x) d x\right. \\
\left\langle\psi_{s, l}^{1}(x) \int_{0}^{1} K(x, t) \varphi_{j_{0}, i}(t) d t d x\right)_{i, l, s}
\end{gathered}
$$

$$
\begin{gathered}
\langle\psi, \psi\rangle-\langle K \psi, \psi\rangle \\
=\left(\int_{0}^{1} \psi_{s, l}(x) \psi_{k, j}(x) d x\right. \\
\left.-\int_{0}^{1} \psi_{s, l}(x) \int_{0}^{1} K(x, t) \psi_{k, j}(t) d t d x\right)_{l, s, k, j}, \\
F_{1}=\int_{0}^{1} f(x) \varphi_{j_{0}, r}(x) d x, \\
F_{2}=\int_{0}^{1} f(x) \psi_{s, l}(x) d x,
\end{gathered}
$$

and the subscripts $i, r, k, j, l$, and $s$ assume values as given below:

$$
\begin{gathered}
i, r=-1, \ldots, 2^{j_{0}}-1, \\
l, k=j_{0}, \ldots, M, \\
s, j=-1, \ldots, 2^{M}-2 .
\end{gathered}
$$

In fact, the entries with significant magnitude are in the $\langle K \varphi, \varphi\rangle-\langle\varphi, \varphi\rangle$ and $\langle K \psi, \psi\rangle-\langle\psi, \psi\rangle$ submatrices which are of order $\left(2^{j_{0}}+1\right)$ and $\left(2^{M+1}+1\right)$, respectively.

3.3. Variational Iteration Method [3-5]. In this section, Fredholm integral equation of second kind given in (7) has been considered for solving (7) by variational iteration method. First, we have to take the partial derivative of (7) with respect to $x$ yielding

$$
Y^{\prime}(x)=f^{\prime}(x)+\int_{0}^{1} K^{\prime}(x, t) y(t) d t .
$$

We apply variation iteration method for (46). According to this method, correction functional can be defined as

$$
\begin{aligned}
y_{n+1}(x) & \\
= & y_{n}(x) \\
& +\int_{0}^{x} \lambda(\xi)\left(y_{n}^{\prime}(\xi)-f^{\prime}(\xi)-\int_{a}^{b} K^{\prime}(\xi, t) \tilde{y}_{n}(t) d t\right) d \xi,
\end{aligned}
$$

where $\lambda(\xi)$ is a general Lagrange multiplier which can be identified optimally by the variational theory, the subscript $n$ denotes the $n$th order approximation, and $\widetilde{y}_{n}$ is considered as a restricted variation; that is, $\delta \tilde{y}_{n}=0$. The successive approximations $y_{n}(x), n \geq 1$ for the solution $y(x)$ can be readily obtained after determining the Lagrange multiplier and selecting an appropriate initial function $y_{0}(x)$. Consequently the approximate solution may be obtained by using

$$
y(x)=\lim _{n \rightarrow \infty} y_{n}(x)
$$


To make the above correction functional stationary, we have

$$
\begin{aligned}
\delta y_{n+1}(x)= & \delta y_{n}(x) \\
& +\delta \int_{0}^{x} \lambda(\xi)\left(y_{n}^{\prime}(\xi)-f^{\prime}(\xi)\right. \\
& \left.-\int_{a}^{b} K^{\prime}(\xi, t) \tilde{y}_{n}(t) d t\right) d \xi \\
= & \delta y_{n}(x)+\int_{0}^{x} \lambda(\xi) \delta\left(y_{n}^{\prime}(\xi)\right) d \xi \\
= & \delta y_{n}(x)+\left.\lambda \delta y_{n}\right|_{\xi=x}-\int_{0}^{x} \lambda^{\prime}(\xi) \delta y_{n}(\xi) d \xi .
\end{aligned}
$$

Under stationary condition,

$$
\delta y_{n+1}=0
$$

implies the following Euler Lagrange equation:

$$
\lambda^{\prime}(\xi)=0
$$

with the following natural boundary condition:

$$
1+\left.\lambda(\xi)\right|_{\xi=x}=0
$$

Solving (51), along with boundary condition (52), we get the general Lagrange multiplier

$$
\lambda=-1 \text {. }
$$

Substituting the identified Lagrange multiplier into (47) results in the following iterative scheme:

$$
\begin{array}{r}
y_{n+1}(x)=y_{n}(x) \\
-\int_{0}^{x}\left(y_{n}^{\prime}(\xi)-f^{\prime}(\xi)-\int_{a}^{b} K^{\prime}(\xi, t) \tilde{y}_{n}(t) d t\right) d \xi, \\
n \geq 0 .
\end{array}
$$

By starting with initial approximate function $y_{0}(x)=f(x)$ (say), we can determine the approximate solution $y(x)$ of (7).

\section{Numerical Methods for System of Linear Fredholm Integral Equations of Second Kind}

Consider the system of linear Fredholm integral equations of second kind of the following form:

$$
\begin{array}{r}
\sum_{j=1}^{n} y_{j}(x)=f_{i}(x)+\sum_{j=1}^{n} \int_{0}^{1} K_{i, j}(x, t) y_{j}(t) d t \\
i=1,2, \ldots, n,
\end{array}
$$

where $f_{i}(x)$ and $K_{i, j}(x, t)$ are known functions and $y_{j}(x)$ are the unknown functions for $i, j=1,2, \ldots, n$.
4.1. Application of Haar Wavelet Method [9]. In this section, an efficient algorithm for solving Fredholm integral equations with Haar wavelets is analyzed. The present algorithm takes the following essential strategy. The Haar wavelet is first used to decompose integral equations into algebraic systems of linear equations, which are then solved by collocation methods.

4.1.1. Haar Wavelets. The compact set of scale functions is chosen as

$$
h_{0}= \begin{cases}1, & 0 \leq x<1 \\ 0, & \text { others. }\end{cases}
$$

The mother wavelet function is defined as

$$
h_{1}(x)= \begin{cases}1, & 0 \leq x<\frac{1}{2}, \\ -1, & \frac{1}{2} \leq x<1, \\ 0, & \text { others. }\end{cases}
$$

The family of wavelet functions generated by translation and dilation of $h_{1}(x)$ are given by

$$
h_{n}(x)=h_{1}\left(2^{j} x-k\right)
$$

where $n=2^{j}+k, j \geq 0,0 \leq k<2^{j}$.

Mutual orthogonalities of all Haar wavelets can be expressed as

$$
\int_{0}^{1} h_{m}(x) h_{n}(x) d x=2^{-j} \delta_{m n}= \begin{cases}2^{-j}, & m=n=2^{j}+k \\ 0, & m \neq n .\end{cases}
$$

4.1.2. Function Approximation. An arbitrary function $y(x) \in$ $L^{2}[0,1)$ can be expanded into the following Haar series:

$$
y(x)=\sum_{n=0}^{+\infty} c_{n} h_{n}(x)
$$

where the coefficients $c_{n}$ are given by

$$
\begin{array}{r}
c_{n}=2^{j} \int_{0}^{1} y(x) h_{n}(x) d x, \\
n=2^{j}+k, \quad j \geq 0, \quad 0 \leq k<2^{j} .
\end{array}
$$

In particular, $c_{0}=\int_{0}^{1} y(x) d x$.

The previously mentioned expression in (60) can be approximately represented with finite terms as follows:

$$
y(x) \approx \sum_{n=0}^{m-1} c_{n} h_{n}(x)=C_{(m)}^{T} h_{(m)}(x),
$$

where the coefficient vector $C_{(m)}^{T}$ and the Haar function vector $h_{(m)}(x)$ are, respectively, defined as

$$
\begin{gathered}
C_{(m)}^{T}=\left[c_{0}, c_{1}, \ldots, c_{m-1}\right], \quad m=2^{j}, \\
h_{(m)}(x)=\left[h_{0}(x), h_{1}(x), \ldots, h_{m-1}(x)\right]^{T}, \quad m=2^{j} .
\end{gathered}
$$


The Haar expansion for function $K(x, t)$ of order $m$ is defined as follows:

$$
K(x, t) \approx \sum_{u=0}^{m-1} \sum_{v=0}^{m-1} a_{u v} h_{v}(x) h_{u}(t)
$$

where $a_{u v}=2^{i+q} \iint_{0}^{1} K(x, t) h_{v}(x) h_{u}(t) d x d t, u=2^{i}+j, v=$ $2^{q}+r, i, q \geq 0$.

From (62) and (64), we obtain

$$
K(x, t) \approx h_{(m)}^{T}(t) K h_{(m)}(x),
$$

where

$$
K=\left(a_{u v}\right)_{m \times m}^{T}
$$

\subsubsection{Operational Matrices of Integration. We define}

$$
H_{(m)}=\left[h_{(m)}\left(\frac{1}{2 m}\right), h_{(m)}\left(\frac{3}{2 m}\right), \ldots, h_{(m)}\left(\frac{2 m-1}{2 m}\right)\right],
$$

where $H_{(1)}=[1], H_{(2)}=\left[\begin{array}{cc}1 & 1 \\ 1 & -1\end{array}\right]$.

Then, for $m=4$, the corresponding matrix can be represented as

$$
\begin{aligned}
H_{(4)} & =\left[h_{(4)}\left(\frac{1}{8}\right), h_{(4)}\left(\frac{3}{8}\right), \ldots, h_{(4)}\left(\frac{7}{8}\right)\right] \\
& =\left[\begin{array}{cccc}
1 & 1 & 1 & 1 \\
1 & 1 & -1 & -1 \\
1 & -1 & 0 & 0 \\
0 & 0 & 1 & -1
\end{array}\right] .
\end{aligned}
$$

The integration of the Haar function vector $h_{(m)}(t)$ is

$$
\begin{array}{r}
\int_{0}^{x} h_{(m)}(t) d t=P_{(m)} h_{(m)}(x), \\
x \in[0,1),
\end{array}
$$

where $P_{(m)}$ is the operational matrix of order $m$, and

$$
\begin{gathered}
P_{(1)}=\left[\frac{1}{2}\right], \\
P_{(m)}=\frac{1}{2 m}\left[\begin{array}{cc}
2 m P_{(m / 2)} & -H_{(m / 2)} \\
H_{(m / 2)}^{-1} & 0
\end{array}\right] .
\end{gathered}
$$

By recursion of the above formula, we obtain

$$
\begin{gathered}
P_{(2)}=\frac{1}{4}\left[\begin{array}{cc}
2 & -1 \\
1 & 0
\end{array}\right], \\
P_{(4)}=\frac{1}{16}\left[\begin{array}{cccc}
8 & -4 & -2 & -2 \\
4 & 0 & -2 & 2 \\
1 & 1 & 0 & 0 \\
1 & -1 & 0 & 0
\end{array}\right], \\
P_{(8)}=\frac{1}{64}\left[\begin{array}{cccccccc}
32 & -16 & -8 & -8 & -4 & -4 & -4 & -4 \\
16 & 0 & -8 & 8 & -4 & -4 & 4 & 4 \\
4 & 4 & 0 & 0 & -4 & 4 & 0 & 0 \\
4 & 4 & 0 & 0 & 0 & 0 & -4 & 4 \\
1 & 1 & 2 & 0 & 0 & 0 & 0 & 0 \\
1 & 1 & -2 & 0 & 0 & 0 & 0 & 0 \\
1 & -1 & 0 & 2 & 0 & 0 & 0 & 0 \\
1 & -1 & 0 & -2 & 0 & 0 & 0 & 0
\end{array}\right] .
\end{gathered}
$$

Therefore, we get

$$
\begin{aligned}
H_{(m)}^{-1}= & \left(\frac{1}{m}\right) H_{(m)}^{T} \\
& \times \operatorname{diag}(1,1,2,2, \underbrace{2^{2}, \ldots, 2^{2}}_{2^{2}}, \ldots, \underbrace{2^{\alpha-1}, \ldots, 2^{\alpha-1}}_{2^{\alpha-1}}),
\end{aligned}
$$

where $m=2^{\alpha}$ and $\alpha$ is a positive integer.

The inner product of two Haar functions can be represented as

$$
\int_{0}^{1} h_{(m)}(t) h_{(m)}^{T}(t) d t=D
$$

where

$$
\begin{gathered}
D=\operatorname{diag}(1,1,1 / 2,1 / 2, \underbrace{1 / 2^{2}, \ldots, 1 / 2^{2}}_{2^{2}}, \ldots, \\
\underbrace{1 / 2^{\alpha-1}, \ldots, 1 / 2^{\alpha-1}}_{2^{\alpha-1}}) .
\end{gathered}
$$

4.1.4. Haar Wavelet Solution for Fredholm Integral Equations System [9]. Consider the following Fredholm integral equations system defined in (55):

$$
\begin{array}{r}
\sum_{j=1}^{m} y_{j}(x)=f_{i}(x)+\sum_{j=1}^{m} \int_{0}^{1} K_{i, j}(x, t) y_{j}(t) d t \\
i=1,2, \ldots, m .
\end{array}
$$

The Haar series of $y_{j}(x)$ and $K_{i, j}(x, t), i=1,2, \ldots, m ; j=$ $1,2, \ldots, m$ are, respectively, expanded as

$$
\begin{gathered}
y_{j}(x) \approx Y_{j}^{T} h_{(m)}(x), \quad j=1,2, \ldots, m, \\
K_{i, j}(x, t) \approx h_{(m)}^{T}(t) K_{i, j} h_{(m)}(x), \\
i, j=1,2, \ldots, m .
\end{gathered}
$$


Substituting (76) into (75), we get

$$
\begin{array}{r}
\sum_{j=1}^{m} Y_{j}^{T} h_{(m)}(x) \\
=f_{i}(x)+\sum_{j=1}^{m} \int_{0}^{1} Y_{j}^{T} h_{(m)}(t) h_{(m)}^{T}(t) K_{i, j} h_{(m)}(x) d t \\
i=1,2, \ldots, m .
\end{array}
$$

From (77) and (73), we get

$$
\begin{array}{r}
\sum_{j=1}^{m} Y_{j}^{T} h_{(m)}(x)=f_{i}(x)+\sum_{j=1}^{m} Y_{j}^{T} D K_{i, j} h_{(m)}(x), \\
i=1,2, \ldots, m .
\end{array}
$$

Interpolating $m$ collocation points, that is, $\left\{x_{i}\right\}_{i=1}^{m}$, in the interval $[0,1]$ leads to the following algebraic system of equations:

$$
\begin{array}{r}
\sum_{j=1}^{m} Y_{j}^{T} h_{(m)}\left(x_{i}\right)=f_{i}\left(x_{i}\right)+\sum_{j=1}^{m} Y_{j}^{T} D K_{i, j} h_{(m)}\left(x_{i}\right), \\
i=1,2, \ldots, m .
\end{array}
$$

Hence, $Y_{j}, j=1,2, \ldots, m$ can be computed by solving the above algebraic system of equations and consequently the solutions $y_{j}(x) \approx Y_{j}^{T} h_{(m)}(x), j=1,2, \ldots, m$.

4.2. Taylor Series Expansion Method. In this section, we present Taylor series expansion method for solving Fredholm integral equations system of second kind [7]. This method reduces the system of integral equations to a linear system of ordinary differential equation. After including boundary conditions, this system reduces to a system of equations that can be solved easily by any usual methods.

Consider the second kind Fredholm integral equations system defined in (55) as follows:

$$
\begin{array}{r}
y_{i}(x)=f_{i}(x)+\sum_{j=1}^{n} \int_{0}^{1} K_{i, j}(x, t) y_{j}(t) d t, \\
i=1,2, \ldots, n, \quad 0 \leq x \leq 1 .
\end{array}
$$

A Taylor series expansion can be made for the solution of $y_{j}(t)$ in the integral equation $(80)$ :

$$
\begin{aligned}
y_{j}(t)= & y_{j}(x)+y_{j}^{\prime}(x)(t-x)+\cdots \\
& +\frac{1}{m !} y_{j}^{(m)}(x)(t-x)^{m}+E(t),
\end{aligned}
$$

where $E(t)$ denotes the error between $y_{j}(t)$ and its Taylor series expansion in (81).

If we use the first $m$ term of Taylor series expansion and neglect the term containing $E(t)$, that is, $\int_{0}^{1} \sum_{j=1}^{n} K_{i, j}(x, t) E(t) d t$, then, substituting (81) for $y_{j}(t)$ into the integral in (80), we have

$$
\begin{aligned}
& y_{i}(x) \approx f_{i}(x) \\
&+\sum_{j=1}^{n} \int_{0}^{1} K_{i, j}(x, t) \sum_{r=0}^{m} \frac{1}{r !}(t-x)^{r} y_{j}^{(r)}(x) d t, \\
& i=1,2, \ldots, n, \\
& y_{i}(x) \approx f_{i}(x) \\
&+\sum_{j=1}^{n} \sum_{r=0}^{m} \frac{1}{r !} y_{j}^{(r)}(x) \int_{0}^{1} K_{i, j}(x, t)(t-x)^{r} d t, \\
& y_{i}(x)-\sum_{j=1}^{n} \sum_{r=0}^{m} \frac{1}{r !} y_{j}^{(r)}(x)\left[\int_{0}^{1} K_{i, j}(x, t)(t-x)^{r} d t\right] \\
& \approx f_{i}(x), \quad i=1,2, \ldots, n .
\end{aligned}
$$

Equation (83) becomes a linear system of ordinary differential equations that we have to solve. For solving the linear system of ordinary differential equations (83), we require an appropriate number of boundary conditions.

In order to construct boundary conditions, we first differentiate $s$ times both sides of (80) with respect to $x$; that is,

$$
\begin{array}{r}
y_{i}^{(s)}(x)=f_{i}^{(s)}(x)+\sum_{j=1}^{n} \int_{0}^{1} K_{i, j}^{(s)}(x, t) y_{j}(t) d t, \\
i=1,2, \ldots, n, \quad s=1,2, \ldots, m,
\end{array}
$$

where $K_{i, j}^{(s)}(x, t)=\partial^{(s)} K_{i, j}(x, t) / \partial x^{(s)}, s=1,2, \ldots, m$.

Applying the mean value theorem for integral in (84), we have

$$
\begin{array}{r}
y_{i}^{(s)}(x)-\left[\sum_{j=1}^{n} \int_{0}^{1} K_{i, j}^{(s)}(x, t) d t\right] y_{j}(x) \approx f_{i}^{(s)}(x), \\
i=1,2, \ldots, n, \quad s=1,2, \ldots, m .
\end{array}
$$

Now (83) combined with (85) becomes a linear system of algebraic equations that can be solved analytically or numerically.

4.3. Block-Pulse Functions for the Solution of Fredholm Integral Equation. In this section, Block-Pulse functions (BPF) have been utilized for the solution of system of Fredholm integral equations [6].

An $m$-set of BPF is defined as follows:

$$
\Phi_{i}(t)= \begin{cases}1, & (i-1) \frac{T}{m} \leq t<i \frac{T}{m} \\ 0, & \text { otherwise }\end{cases}
$$

with $t \in[0, T), T / m=h$ and $i=1,2, \ldots, m$. 


\subsubsection{Properties of $B P F$}

(1) Disjointness. One has

$$
\Phi_{i}(t) \Phi_{j}(t)= \begin{cases}\Phi_{i}(t), & i=j \\ 0, & i \neq j\end{cases}
$$

$, i, j=1,2, \ldots, m$. This property is obtained from definition of BPF.

(2) Orthogonality. One has

$$
\int_{0}^{T} \Phi_{i}(t) \Phi_{j}(t) d t= \begin{cases}h, & i=j \\ 0, & i \neq j,\end{cases}
$$

$t \in[0, T), i, j=1,2, \ldots, m$. This property is obtained from the disjointness property.

(3) Completeness. For every $f \in L^{2},\{\Phi\}$ is complete; if $\int \Phi f=$ 0 then $f=0$ almost everywhere. Because of completeness of $\{\Phi\}$, we have

$$
\int_{0}^{T} f^{2}(t) d t=\sum_{i=1}^{\infty} f_{i}^{2}\left\|\Phi_{i}(t)\right\|^{2}
$$

for every real bounded function $f(t)$ which is square integrable in the interval $t \in[0, T)$ and $f_{i}=(1 / h) f(t) \Phi_{i}(t) d t$.

4.3.2. Function Approximation. The orthogonality property of BPF is the basis of expanding functions into their BlockPulse series. For every $f(t) \in L^{2}(R)$,

$$
f(t)=\sum_{i=1}^{m} f_{i} \Phi_{i}(t)
$$

where $f_{i}$ is the coefficient of Block-Pulse function, with respect to $i$ th Block-Pulse function $\Phi_{i}(t)$.

The criterion of this approximation is that mean square error between $f(t)$ and its expansion is minimum

$$
\varepsilon=\frac{1}{T} \int_{0}^{T}\left(f(t)-\sum_{j=1}^{m} f_{j} \Phi_{j}(t)\right)^{2} d t
$$

so that we can evaluate Block-Pulse coefficients.

$$
\begin{gathered}
\text { Now } \frac{\partial \varepsilon}{\partial f_{i}}=-\frac{2}{T} \int_{0}^{T}\left(f(t)-\sum_{j=1}^{m} f_{j} \Phi_{j}(t)\right) \Phi_{i}(t) d t=0, \\
\Longrightarrow f_{i}=\frac{1}{h} \int_{0}^{T} f(t) \Phi_{i}(t) d t \quad \text { (using orthogonal property). }
\end{gathered}
$$

In the matrix form, we obtain the following from (90) as follow:

$$
\begin{gathered}
f(t)=\sum_{i=1}^{m} f_{i} \Phi_{i}(t)=F^{T} \Phi(t)=\Phi^{T} F \\
\text { where } F=\left[f_{1}, f_{2}, \ldots, f_{m}\right]^{T}, \\
\Phi(t)=\left[\Phi_{1}(t), \Phi_{2}(t), \ldots, \Phi_{m}(t)\right]^{T} .
\end{gathered}
$$

Now let $K(t, s)$ be two-variable function defined on $t \in[0, T)$ and $s \in[0,1)$; then $K(t, s)$ can be expanded to BPF as

$$
K(t, s)=\Phi^{T}(t) K \Psi(s),
$$

where $\Phi(t)$ and $\Psi(s)$ are $m_{1}$ and $m_{2}$ dimensional Block-Pulse function vectors and $k$ is a $m_{1} \times m_{2}$ Block-Pulse coefficient matrix.

There are two different cases of multiplication of two BPF. The first case is

$$
\Phi(t) \Phi^{T}(t)=\left(\begin{array}{cccc}
\Phi_{1}(t) & 0 & \cdots & 0 \\
0 & \Phi_{2}(t) & \cdots & 0 \\
\vdots & \vdots & \ddots & \vdots \\
0 & 0 & \cdots & \Phi_{m}(t)
\end{array}\right) .
$$

It is obtained from disjointness property of BPF. It is a diagonal matrix with $m$ Block-Pulse functions.

The second case is

$$
\Phi^{T}(t) \Phi(t)=1
$$

because $\sum_{i=1}^{m}\left(\Phi_{i}(t)\right)^{2}=\sum_{i=1}^{m} \Phi_{i}(t)=1$.

Operational Matrix of Integration. BPF integration property can be expressed by an operational equation as

$$
\int_{0}^{T} \Phi(t) d t=P \Phi(t)
$$

where

$$
\Phi(t)=\left[\Phi_{1}(t), \Phi_{2}(t), \ldots, \Phi_{m}(t)\right]^{T} .
$$

A general formula for $P_{m \times m}$ can be written as

$$
P=\frac{1}{2}\left(\begin{array}{ccccc}
1 & 2 & 2 & \cdots & 2 \\
0 & 1 & 2 & \cdots & 2 \\
0 & 0 & 1 & \cdots & 2 \\
\vdots & \vdots & \vdots & \ddots & \vdots \\
0 & 0 & 0 & \cdots & 1
\end{array}\right) .
$$

By using this matrix, we can express the integral of a function $f(t)$ into its Block-Pulse series

$$
\int_{0}^{t} f(t) d t=\int_{0}^{t} F^{T} \Phi(t) d t=F^{T} P \Phi(t) .
$$

4.3.3. Solution for Linear Integral Equations System. Consider the integral equations system from (55) as follows:

$$
\begin{array}{r}
\sum_{j=1}^{n} y_{j}(x)=f_{i}(x)+\sum_{j=1}^{n} \int_{\alpha}^{\beta} K_{i, j}(x, t) y_{j}(t) d t \\
i=1,2, \ldots, n .
\end{array}
$$

Block-Pulse coefficients of $y_{j}(x), j=1,2, \ldots, n$ in the interval $x \in[\alpha, \beta)$ can be determined from the known functions $f_{i}(x), i=1,2, \ldots, n$ and the kernels $K_{i, j}(x, t), i, j=1,2, \ldots n$. Usually, we consider $\alpha=0$ to facilitie the use of Block-Pulse 
functions. In case $\alpha \neq 0$, we set $X=((x-\alpha) /(\beta-\alpha)) T$, where $T=m h$.

We approximate $f_{i}(x), y_{j}(x), K_{i, j}(x, t)$ by its BPF as follows:

$$
\begin{gathered}
f_{i}(x) \approx F_{i}^{T} \Phi(x), \\
y_{j}(x) \approx Y_{j}^{T} \Phi(x), \\
K_{i, j}(x, t) \approx \Phi^{T}(t) K_{i, j} \Phi(x),
\end{gathered}
$$

where $F_{i}, Y_{j}$, and $K_{i, j}$ are defined in Section 4.3.2, and substituting (102) into (101), we have

$$
\begin{gathered}
\sum_{j=1}^{n} Y_{j}^{T} \Phi(x)=F_{i}^{T} \Phi(x) \\
+\sum_{j=1}^{n} \int_{0}^{m h} Y_{j}^{T} \Phi(t) \Phi^{T}(t) K_{i, j} \Phi(x) d t, \\
i=1,2, \ldots, n, \\
\sum_{j=1}^{n} Y_{j}^{T} \Phi(x)=F_{i}^{T} \Phi(x)+\sum_{j=1}^{n} Y_{j}^{T} h I K_{i, j} \Phi(x), \\
i=1,2, \ldots, n,
\end{gathered}
$$

since

$$
\int_{0}^{m h} \Phi(t) \Phi^{T}(t) d t=h I
$$

From (104), we get

$$
\sum_{j=1}^{n}\left(I-h K_{i, j}^{T}\right) Y_{j}=F_{i}, \quad i=1,2, \ldots, n .
$$

Set $A_{i, j}=I-h K_{i, j}^{T}$; then we have from (106)

$$
\sum_{j=1}^{n} A_{i, j} Y_{j}=F_{i}, \quad i=1,2, \ldots, n
$$

which is a linear system

$$
\left(\begin{array}{ccccc}
A_{11} & A_{12} & \cdot & \cdot & A_{1 n} \\
A_{21} & A_{22} & \cdot & \cdot & A_{2 n} \\
\cdot & \cdot & \cdot & & \cdot \\
\cdot & \cdot & \cdot & \cdot \\
\cdot & \cdot & & \cdot & \cdot \\
A_{n 1} & A_{n 2} & \cdot & \cdot & A_{n n}
\end{array}\right)\left(\begin{array}{c}
Y_{1} \\
Y_{2} \\
\cdot \\
\cdot \\
\cdot \\
Y_{n}
\end{array}\right)=\left(\begin{array}{c}
F_{1} \\
F_{2} \\
\cdot \\
\cdot \\
\cdot \\
F_{n}
\end{array}\right)
$$

After solving the above system we can find $Y_{j}, j=1,2, \ldots, n$ and hence obtain the solutions $y_{j}=\Phi^{T} Y_{j}, j=1,2, \ldots, n$.

\section{Numerical Methods for Nonlinear Fredholm-Hammerstein Integral Equation}

We consider the second kind nonlinear Fredholm integral equation of the following form:

$$
\begin{gathered}
u(x)=f(x)+\int_{0}^{1} K(x, t) F(t, u(t)) d t, \\
0 \leq x \leq 1,
\end{gathered}
$$

where $K(x, t)$ is the kernel of the integral equation, $f(x)$ and $K(x, t)$ are known functions, and $u(x)$ is the unknown function that is to be determined.

5.1. B-Spline Wavelet Method. In this section, nonlinear Fredholm integral equation of second kind of the form given in (109) has been solved by using $B$-spline wavelets [11].

$B$-spline scaling and wavelet functions in the interval $[0,1]$ and function approximation have been defined in Sections 3.1.1 and 3.1.2, respectively.

First, we assume that

$$
\begin{gathered}
y(x)=F(x, u(x)), \\
0 \leq x \leq 1 .
\end{gathered}
$$

Now, from (16), we can approximate the functions $u(x)$ and $y(x)$ as

$$
\begin{aligned}
& u(x)=A^{T} \Psi(x), \\
& y(x)=B^{T} \Psi(x),
\end{aligned}
$$

where $A$ and $B$ are $\left(2^{M+1}+m-1\right) \times 1$ column vectors similar to $C$ defined in (17).

Again, by using dual of the wavelet functions, we can approximate the functions $f(x)$ and $K(x, t)$ as follows:

$$
\begin{gathered}
F(x)=D^{T} \widetilde{\Psi}(x), \\
K(x, t)=\widetilde{\Psi}^{T}(t) \Theta \widetilde{\Psi}(x),
\end{gathered}
$$

where

$$
\Theta_{(i, j)}=\int_{0}^{1}\left[\int_{0}^{1} K(x, t) \Psi_{i}(t) d t\right] \Psi_{j}(x) d x .
$$

From (110)-(112), we get

$$
\begin{aligned}
\int_{0}^{1} K(x, t) F(t, u(t)) d t \\
\quad=\int_{0}^{1} B^{T} \Psi(t) \widetilde{\Psi}^{T}(t) \Theta \widetilde{\Psi}(x) d t \\
=B^{T}\left[\int_{0}^{1} \Psi(t) \widetilde{\Psi}^{T}(t) d t\right] \Theta \widetilde{\Psi}(x) \\
=B^{T} \Theta \widetilde{\Psi}(x), \quad \text { since } \int_{0}^{1} \Psi(t) \widetilde{\Psi}^{T}(t) d t=I .
\end{aligned}
$$


Applying (110)-(114) in (109), we get

$$
A^{T} \Psi(x)=D^{T} \widetilde{\Psi}(x)+B^{T} \Theta \widetilde{\Psi}(x) .
$$

Multiplying (115) by $\Psi^{T}(x)$ both sides from the right and integrating both sides with respect to $x$ from 0 to 1 , we have

$$
\begin{gathered}
A^{T} P=D^{T}+B^{T} \Theta, \\
A^{T} P-D^{T}-B^{T} \Theta=0,
\end{gathered}
$$

where $P$ is a $\left(2^{M+1}+m-1\right) \times\left(2^{M+1}+m-1\right)$ square matrix given by

$$
\begin{gathered}
P=\int_{0}^{1} \Psi(x) \Psi^{T}(x) d x=\left[\begin{array}{ll}
P_{1} & \\
& P_{2}
\end{array}\right], \\
\int_{0}^{1} \widetilde{\Psi}(x) \Psi^{T}(x) d x=I .
\end{gathered}
$$

Equation (116) gives a system of $\left(2^{M+1}+m-1\right)$ algebraic equations with $2\left(2^{M+1}+m-1\right)$ unknowns for $A$ and $B$ vectors given in (111).

To find the solution $u(x)$ in (111), we first utilize the following equation:

$$
F\left(x, A^{T} \Psi(x)\right)=B^{T} \Psi(x),
$$

with the collocation points $x_{i}=(i-1) /\left(2^{M+1}+m-2\right)$, where $i=1,2, \ldots, 2^{M+1}+m-1$.

Equation (118) gives a system of $\left(2^{M+1}+m-1\right)$ algebraic equations with $2\left(2^{M+1}+m-1\right)$ unknowns, for $A$ and $B$ vectors given in (111).

Combining (116) and (118), we have a total of $2\left(2^{M+1}+\right.$ $m-1)$ system of algebraic equations with $2\left(2^{M+1}+m-\right.$ 1) unknowns for $A$ and $B$. Solving those equations for the unknown coefficients in the vectors $A$ and $B$, we can obtain the solution $u(x)=A^{T} \Psi(x)$.

5.2. Quadrature Method Applied to Fredholm Integral Equation. In this section, Quadrature method has been applied to solve nonlinear Fredholm-Hammerstein integral equation [10].

The quadrature methods like Simpson rule and modified trapezoid method are applied for solving a definite integral as follows.

\subsubsection{Simpson's Rule. One has}

$$
\begin{aligned}
\int_{a}^{b} f(x) d x & =\sum_{i=1}^{n-1} \int_{x_{i-1}}^{x_{i+1}} f(x) d x \\
& =\frac{h}{3} f(a)+\frac{4 h}{3} \sum_{i=1}^{n / 2} f\left(x_{2 i-1}\right)
\end{aligned}
$$

$$
\begin{aligned}
& +\frac{2 h}{3} \sum_{i=1}^{(n-1) / 2} f\left(x_{2 i}\right) \\
& +\frac{h}{3} f(b) \\
& -\frac{(b-a)}{180} h^{4} f^{(4)}(\eta) .
\end{aligned}
$$

5.2.2. Modified Trapezoid Rule. One has

$$
\begin{aligned}
\int_{a}^{b} f(x) d x= & \sum_{i=1}^{n} \int_{x_{i-1}}^{x_{i}} f(x) d x \\
= & \frac{h}{2} f(a)+h \sum_{i=1}^{n-1} f\left(x_{i}\right) \\
& +\frac{h}{2} f(b) \\
& +\frac{h^{2}}{12}\left[f^{\prime}(a)-f^{\prime}(b)\right] .
\end{aligned}
$$

Consider the nonlinear Fredholm integral equation of second kind defined in (109) as follows:

$$
\begin{array}{r}
u(x)=f(x)+\int_{a}^{b} K(x, t) F(u(t)) d t, \\
a \leq x \leq b .
\end{array}
$$

For solving (121), we approximate the right-hand integral of (121) with Simpson's rule and modified trapezoid rule; then we get the following.

5.2.3. Simpson's Rule. One has

$$
\begin{aligned}
u(x)=f(x) & \\
+ & \frac{h}{3}\left[K\left(x, t_{0}\right) F\left(u_{0}\right)\right. \\
& +4 \sum_{j=1}^{n / 2} K\left(x, t_{2 j-1}\right) F\left(u_{2 j-1}\right) \\
& +2 \sum_{j=1}^{(n / 2)-1} K\left(x, t_{2 j}\right) F\left(u_{2 j}\right) \\
& \left.+K\left(x, t_{n}\right) F\left(u_{n}\right)\right] .
\end{aligned}
$$

Hence, for $x=x_{0}, x_{1}, \ldots, x_{n}$ and $t=t_{0}, t_{1}, \ldots, t_{n}$ in (122), we have

$$
\begin{aligned}
& u\left(x_{i}\right)=f\left(x_{i}\right) \\
&+\frac{h}{3}\left[K\left(x_{i}, t_{0}\right) F\left(u_{0}\right)\right. \\
&+4 \sum_{j=1}^{n / 2} K\left(x_{i}, t_{2 j-1}\right) F\left(u_{2 j-1}\right)
\end{aligned}
$$




$$
\begin{aligned}
& +2 \sum_{j=1}^{(n / 2)-1} K\left(x_{i}, t_{2 j}\right) F\left(u_{2 j}\right) \\
& \left.+K\left(x_{i}, t_{n}\right) F\left(u_{n}\right)\right] .
\end{aligned}
$$

Equation (123) is a nonlinear system of equations and, by solving (123), we obtain the unknowns $u\left(x_{i}\right)$ for $i=0,1, \ldots, n$.

5.2.4. Modified Trapezoid Rule. One has

$$
\begin{aligned}
u(x)= & f(x) \\
& +\frac{h}{2} K\left(x, t_{0}\right) F\left(u_{0}\right) \\
& +h \sum_{j=1}^{n-1} K\left(x, t_{j}\right) F\left(u_{j}\right) \\
+ & \frac{h}{2} K\left(x, t_{n}\right) F\left(u_{n}\right) \\
& +\frac{h^{2}}{12}\left[J\left(x, t_{0}\right) F\left(u_{0}\right)\right. \\
& +K\left(x, t_{0}\right) u_{0}^{\prime} F^{\prime}\left(u_{0}\right) \\
& -J\left(x, t_{n}\right) F\left(u_{n}\right) \\
& \left.-K\left(x, t_{n}\right) u_{n}^{\prime} F^{\prime}\left(u_{n}\right)\right],
\end{aligned}
$$

where $J(x, t)=\partial K(x, t) / \partial t$. have

For $x=x_{0}, x_{1}, \ldots, x_{n}$ and $t=t_{0}, t_{1}, \ldots, t_{n}$ in (124), we

$$
\begin{aligned}
u\left(x_{i}\right)= & f\left(x_{i}\right) \\
& +\frac{h}{2} K\left(x_{i}, t_{0}\right) F\left(u_{0}\right) \\
& +h \sum_{j=1}^{n-1} K\left(x_{i}, t_{j}\right) F\left(u_{j}\right) \\
+ & \frac{h}{2} K\left(x_{i}, t_{n}\right) F\left(u_{n}\right) \\
+ & \frac{h^{2}}{12}\left[J\left(x_{i}, t_{0}\right) F\left(u_{0}\right)\right. \\
& +K\left(x_{i}, t_{0}\right) u_{0}^{\prime} F^{\prime}\left(u_{0}\right) \\
& -J\left(x_{i}, t_{n}\right) F\left(u_{n}\right) \\
& \left.-K\left(x_{i}, t_{n}\right) u_{n}^{\prime} F^{\prime}\left(u_{n}\right)\right],
\end{aligned}
$$

for $i=0,1, \ldots, n$.
This is a system of $(n+1)$ equations and $(n+3)$ unknowns. By taking derivative from (121) and setting $H(x, t)=$ $\partial K(x, t) / \partial x$, we obtain

$$
u^{\prime}(x)=f^{\prime}(x)+\int_{a}^{b} H(x, t) F(u(t)) d t
$$

$$
a \leq x \leq b .
$$

If $u$ is a solution of (121), then it is also solution of (126). By using trapezoid rule for (126) and replacing $x=x_{i}$, we get

$$
\begin{aligned}
u^{\prime}\left(x_{i}\right)= & f^{\prime}\left(x_{i}\right) \\
& +\frac{h}{2} H\left(x_{i}, t_{0}\right) F\left(u_{0}\right) \\
& +h \sum_{j=1}^{n-1} H\left(x_{i}, t_{j}\right) F\left(u_{j}\right) \\
& +\frac{h}{2} H\left(x_{i}, t_{n}\right) F\left(u_{n}\right),
\end{aligned}
$$

for $i=0,1, \ldots, n$. In case of $i=0, n$ from system (127), we obtain two equations.

Now (127) combined with (125) generates the nonlinear system of equations as follows:

$$
\begin{aligned}
u\left(x_{i}\right)= & \left(\frac{h}{2} K\left(x_{i}, t_{0}\right)+\frac{h^{2}}{12} J\left(x_{i}, t_{0}\right)\right) F\left(u_{0}\right) \\
& +h \sum_{j=1}^{n-1} K\left(x_{i}, t_{j}\right) F\left(u_{j}\right) \\
& +\left(\frac{h}{2} K\left(x_{i}, t_{n}\right)-\frac{h^{2}}{12} J\left(x_{i}, t_{n}\right)\right) F\left(u_{n}\right) \\
& +\frac{h^{2}}{12}\left(K\left(x_{i}, t_{0}\right) u_{0}^{\prime} F^{\prime}\left(u_{0}\right)\right. \\
u^{\prime}\left(x_{0}\right)= & f^{\prime}\left(x_{0}\right) \\
& +\frac{h}{2} H\left(x_{0}, t_{0}\right) F\left(u_{0}\right) \\
& +\frac{h}{n-1} H\left(x_{j=1}^{n} H\left(x_{0}, t_{j}\right) F\left(u_{j}\right)\right. \\
& +\frac{h}{2} H\left(x_{0}, t_{n}\right) F\left(u_{n}\right),
\end{aligned}
$$




$$
\begin{aligned}
u^{\prime}\left(x_{n}\right)= & f^{\prime}\left(x_{n}\right) \\
& +\frac{h}{2} H\left(x_{n}, t_{0}\right) F\left(u_{0}\right) \\
& +h \sum_{j=1}^{n-1} H\left(x_{n}, t_{j}\right) F\left(u_{j}\right) \\
& +\frac{h}{2} H\left(x_{n}, t_{n}\right) F\left(u_{n}\right) .
\end{aligned}
$$

By solving this system with $(n+3)$ nonlinear equations and $(n+3)$ unknowns, we can obtain the solution of $(109)$.

5.3. Wavelet Galerkin Method. In this section, the continuous Legendre wavelets [12], constructed on the interval [0,1], are applied to solve the nonlinear Fredholm integral equation of the second kind. The nonlinear part of the integral equation is approximated by Legendre wavelets, and the nonlinear integral equation is reduced to a system of nonlinear equations.

We have the following family of continuous wavelets with dilation parameter $a$ and the translation parameter $b$

$$
\begin{array}{r}
\psi_{a, b}(t)=|a|^{-1 / 2} \psi\left(\frac{t-b}{a}\right), \\
a, b \in R, \quad a \neq 0 .
\end{array}
$$

Legendre wavelets $\psi_{m, n}(t)=\psi(k, \widehat{n}, m, t)$ have four arguments; $k=2,3, \ldots, \widehat{n}=2 n-1, n=1,2, \ldots, 2^{k-1}, m$ is the order for Legendre polynomials and $t$ is the normalized time.

Legendre wavelets are defined on $[0,1)$ by

$$
\begin{aligned}
& \psi_{m, n}(t) \\
& = \begin{cases}\left(m+\frac{1}{2}\right)^{1 / 2} 2^{k / 2} L_{m}\left(2^{k} t-\widehat{n}\right), & \frac{\widehat{n}-1}{2^{k}} \leq t<\frac{\widehat{n}+1}{2^{k}} \\
0, & \text { otherwise, }\end{cases}
\end{aligned}
$$

where $L_{m}(t)$ are the well-known Legendre polynomials of order $m$, which are orthogonal with respect to the weight function $w(t)=1$ and satisfy the following recursive formula:

$$
\begin{gathered}
L_{0}(t)=1, \\
L_{1}(t)=t, \\
L_{m+1}(t)=\frac{2 m+1}{m+1} t L_{m}(t) \\
-\frac{m}{m+1} L_{m-1}(t), \quad m=1,2,3, \ldots
\end{gathered}
$$

The set of Legendre wavelets are an orthonormal set.

5.3.1. Function Approximation. A function $f(x) \in L^{2}[0,1]$ can be expanded as

$$
f(x)=\sum_{n=1}^{\infty} \sum_{m=0}^{\infty} c_{n, m} \psi_{n, m}(x),
$$

where

$$
c_{n, m}=\left\langle f(x), \psi_{n, m}(x)\right\rangle .
$$

If the infinite series in (132) is truncated, then (132) can be written as

$$
f(x) \approx \sum_{n=1}^{2^{k-1}} \sum_{m=0}^{M-1} c_{n, m} \psi_{n, m}(x)=C^{T} \Psi(x)
$$

where $C$ and $\Psi(x)$ are $2^{k-1} M \times 1$ matrices given by

$$
\begin{gathered}
C=\left[c_{1,0}, c_{1,1}, \ldots, c_{1, M-1}, c_{2,0}, \ldots,\right. \\
\left.c_{2, M-1}, \ldots, c_{2^{k-1}, 0}, \ldots, c_{2^{k-1}, M-1}\right]^{T}, \\
\Psi(x)=\left[\psi_{1,0}(x), \ldots, \psi_{1, M-1}(x),\right. \\
\psi_{2,0}(x), \ldots, \psi_{2, M-1}(x), \ldots, \\
\left.\psi_{2^{k-1}, 0}(x), \ldots, \psi_{2^{k-1}, M-1}(x)\right]^{T} .
\end{gathered}
$$

Similarly, a function $k(x, t) \in L^{2}([0,1] \times[0,1])$ can be approximated as

$$
k(x, t) \approx \Psi^{T}(t) K \Psi(x)
$$

where $K$ is $\left(2^{k-1} M \times 2^{k-1} M\right)$ matrix, with

$$
K_{i, j}=\left\langle\psi_{i}(t),\left\langle k(x, t), \psi_{j}(x)\right\rangle\right\rangle .
$$

Also, the integer power of a function can be approximated as

$$
[y(x)]^{p}=\left[Y^{T} \Psi(x)\right]^{p}=Y_{p}^{* T} \Psi(x),
$$

where $Y_{p}^{*}$ is a column vector, whose elements are nonlinear combinations of the elements of the vector $Y . Y_{p}^{*}$ is called the operational vector of the $p$ th power of the function $y(x)$.

5.3.2. The Operational Matrices. The integration of the vector $\Psi(x)$ defined in (136) can be obtained as

$$
\int_{0}^{t} \Psi\left(t^{\prime}\right) d t^{\prime}=P \Psi(t)
$$

where $P$ is the $\left(2^{k-1} M \times 2^{k-1} M\right)$ operational matrix for integration and is given in [23] as

$$
P=\left[\begin{array}{ccccc}
L & H & \cdots & H & H \\
0 & L & \cdots & H & H \\
\vdots & \vdots & \ddots & \vdots & \vdots \\
0 & 0 & \cdots & L & H \\
0 & 0 & \cdots & 0 & L
\end{array}\right]
$$

In (141), $H$ and $L$ are $(M \times M)$ matrices given in [23] as 


$$
\begin{aligned}
& H=\frac{1}{2^{k}}\left[\begin{array}{cccc}
2 & 0 & \cdots & 0 \\
0 & 0 & \cdots & 0 \\
\vdots & \vdots & \ddots & \vdots \\
0 & 0 & \cdots & 0
\end{array}\right] \\
& L=\frac{1}{2^{k}}\left[\begin{array}{ccccccc}
1 & \frac{1}{\sqrt{3}} & 0 & 0 & \cdots & 0 & 0 \\
-\frac{\sqrt{3}}{3} & 0 & \frac{\sqrt{3}}{3 \sqrt{5}} & 0 & \cdots & 0 & 0 \\
0 & -\frac{\sqrt{5}}{5 \sqrt{3}} & 0 & \frac{\sqrt{5}}{5 \sqrt{7}} & \cdots & 0 & 0 \\
0 & 0 & -\frac{\sqrt{7}}{7 \sqrt{5}} & 0 & \cdots & 0 & 0 \\
\vdots & \vdots & \vdots & \vdots & \ddots & 0 & \vdots \\
0 & 0 & 0 & 0 & \cdots & \frac{-\sqrt{2 M-1}}{2 M-3) \sqrt{2 M-1}} \\
0 & 0 & 0 & 0 & \cdots & \frac{-\sqrt{2 M-1) \sqrt{2 M-3}}}{(2 M-1)} & 0
\end{array}\right] .
\end{aligned}
$$

The integration of the product of two Legendre wavelets vector functions is obtained as

$$
\int_{0}^{1} \Psi(t) \Psi^{T}(t) d t=I
$$

where $I$ is an identity matrix.

The product of two Legendre wavelet vector functions is defined as

$$
\Psi(t) \Psi^{T}(t) C=\widetilde{C}^{T} \Psi(t),
$$

where $C$ is a vector given in (135) and $\widetilde{C}$ is $\left(2^{k-1} M \times 2^{k-1} M\right)$ matrix, which is called the product operation of Legendre wavelet vector functions $[23,24]$.

5.3.3. Solution of Fredholm Integral Equation of Second Kind. Consider the nonlinear Fredholm-Hammerstein integral equation of second kind of the form

$$
y(x)=f(x)+\int_{0}^{1} k(x, t)[y(t)]^{p} d t
$$

where $f \in L^{2}[0,1], k \in L^{2}([0,1] \times[0,1]), y$ is an unknown function, and $p$ is a positive integer.

We can approximate the following functions as

$$
\begin{gathered}
f(x) \approx F^{T} \Psi(x), \\
y(x) \approx Y^{T} \Psi(x), \\
k(x, t) \approx \Psi^{T}(t) K \Psi(x), \\
{[y(x)]^{p} \approx Y^{* T} \Psi(x) .}
\end{gathered}
$$

Substituting (146) into (145), we have

$$
\begin{aligned}
Y^{T} \Psi(x)= & F^{T} \Psi(x) \\
& +\int_{0}^{1} Y^{* T} \Psi(t) \Psi^{T}(t) K \Psi(x) d t \\
= & F^{T} \Psi(x) \\
& +Y^{* T}\left(\int_{0}^{1} \Psi(t) \Psi^{T}(t) d t\right) K \Psi(x) \\
= & F^{T} \Psi(x)+Y^{* T} K \Psi(x) \\
= & \left(F^{T}+Y^{* T} K\right) \Psi(x) \\
\Longrightarrow & Y^{T}-Y^{* T} K-F^{T}=0 .
\end{aligned}
$$

Equation (147) is a system of algebraic equations. Solving (147), we can obtain the solution $y(x) \approx Y^{T} \Psi(x)$.

5.4. Homotopy Perturbation Method. Consider the following nonlinear Fredholm integral equation of second kind of the form

$$
\begin{array}{r}
u(x)=f(x)+\int_{0}^{1} K(x, t) F(u(t)) d t \\
0 \leq x \leq 1 .
\end{array}
$$

For solving (148) by Homotopy perturbation method (HPM) [14-16], we consider (148) as

$$
L(u)=u(x)-f(x)-\int_{0}^{1} K(x, t) F(u(t)) d t=0 .
$$


As a possible remedy, we can define $H(u, p)$ by

$$
\begin{aligned}
& H(u, 0)=N(u), \\
& H(u, 1)=L(u),
\end{aligned}
$$

where $N(u)$ is an integral operator with known solution $u_{0}$.

We may choose a convex homotopy by

$$
H(u, p)=(1-p) N(u)+p L(u)=0
$$

and continuously trace an implicitly defined curve from a starting point $H\left(u_{0}, 0\right)$ to a solution function $H(U, 1)$. The embedding parameter $p$ monotonically increases from zero to unit as the trivial problem $L(u)=0$. The embedding parameter $p \in(0,1]$ can be considered as an expanding parameter. The HPM uses the homotopy parameter $p$ as an expanding parameter; that is,

$$
u=u_{0}+p u_{1}+p^{2} u_{2}+\cdots .
$$

When $p \rightarrow 1$, (152a) corresponding to (151) become the approximate solution of (149) as follows:

$$
U=\lim _{p \rightarrow 1} u=u_{0}+u_{1}+u_{2}+\cdots .
$$

The series in (152b) converges in most cases, and the rate of convergence depends on $L(u)[14]$.

Consider

$$
N(u)=u(x)-f(x) .
$$

The nonlinear term $F(u)$ can be expressed in He polynomials [25] as

$$
\begin{aligned}
F(u)= & \sum_{m=0}^{\infty} p^{m} H_{m}\left(u_{0}, u_{1}, \ldots, u_{m}\right) \\
= & H_{0}\left(u_{0}\right)+p H_{1}\left(u_{0}, u_{1}\right) \\
& +\cdots+p^{m} H_{m}\left(u_{0}, u_{1}, \ldots u_{m}\right)+\cdots,
\end{aligned}
$$

where

$$
\begin{aligned}
& H_{m}\left(u_{0}, u_{1}, \ldots, u_{m}\right) \\
& \quad=\left.\frac{1}{m !} \frac{\partial^{m}}{\partial p^{m}}\left(F\left(\sum_{k=0}^{m} p^{k} u_{k}\right)\right)\right|_{p=0}, \quad m \geq 0 .
\end{aligned}
$$

Substituting (152a), (153), and (154) into (151), we have

$$
\begin{aligned}
& (1-p)\left(\left(u_{0}+p u_{1}+\cdots\right)-f(x)\right) \\
& +p\left(\left(u_{0}+p u_{1}+\cdots\right)-f(x)\right. \\
& \left.\quad-\int_{0}^{1} K(x, t) \sum_{m=0}^{\infty} p^{m} H_{m}\left(u_{0}, u_{1}, \ldots, u_{m}\right) d t\right)=0 \\
& \Longrightarrow\left(u_{0}+p u_{1}+\cdots\right)-f(x) \\
& -p \int_{0}^{1} K(x, t) \sum_{m=0}^{\infty} p^{m} H_{m}\left(u_{0}, u_{1}, \ldots, u_{m}\right) d t=0 .
\end{aligned}
$$

Equating the terms with identical power of $p$ in (156), we have

$$
\begin{gathered}
p^{0}: u_{0}(x)-f(x)=0 \Longrightarrow u_{0}(x)=f(x) \\
p^{1}: u_{1}(x)-\int_{0}^{1} K(x, t) H_{0} d t=0 \Longrightarrow u_{1}(x) \\
=\int_{0}^{1} K(x, t) H_{0} d t \\
p^{2}: u_{2}(x)-\int_{0}^{1} K(x, t) H_{1} d t=0 \Longrightarrow u_{2}(x) \\
=\int_{0}^{1} K(x, t) H_{1} d t
\end{gathered}
$$

and in general form we have

$$
\begin{gathered}
u_{0}(x)=f(x) \\
u_{n+1}(x)=\int_{0}^{1} K(x, t) H_{n} d t, \quad n=0,1,2, \ldots
\end{gathered}
$$

Hence, we can obtain the approximate solution of aforesaid equation (148) from (152b).

5.5. Adomian Decomposition Method. Adomian decomposition method (ADM) [16-18] has been applied to a wide class of functional equations. This method gives the solution as an infinite series usually converging to an accurate solution. Let us consider the nonlinear Fredholm integral equation of second kind as follows:

$$
\begin{array}{r}
u(x)=f(x)+\int_{a}^{b} K(x, t)(L u(t)+N u(t)) d t \\
a \leq x \leq b,
\end{array}
$$

where $L(u(t))$ and $N(u(t))$ are the linear and nonlinear terms, respectively.

The Adomian decomposition method (ADM) consists of representing $u(x)$ as a series

$$
u(x)=\sum_{m=0}^{\infty} u_{m}(x)
$$

In the view of $\mathrm{ADM}$, the nonlinear term $N u$ can be represented as

$$
\begin{gathered}
N u=\sum_{n=0}^{\infty} A_{n} \\
\text { where } A_{n}=\left.\frac{1}{n !}\left(\frac{\partial^{n}}{\partial \lambda^{n}} N\left(\sum_{k=0}^{\infty} \lambda^{k} u_{k}\right)\right)\right|_{\lambda=0} .
\end{gathered}
$$


Now substituting (160) and (161) into (159), we have

$$
\begin{aligned}
\sum_{m=0}^{\infty} u_{m}(x)= & f(x) \\
& +\int_{a}^{b} K(x, t)\left(L\left(\sum_{m=0}^{\infty} u_{m}(t)\right)+\sum_{m=0}^{\infty} A_{m}\right) d t
\end{aligned}
$$

and, then, ADM uses the recursive relations

$$
\begin{gathered}
u_{0}(x)=f(x), \\
u_{m}(x)=\int_{a}^{b} K(x, t)\left(L\left(u_{m-1}(t)\right)+A_{m-1}(t)\right) d t \\
m \geq 1,
\end{gathered}
$$

where $A_{m}$ is so-called Adomian polynomial.

Therefore, we obtain the $n$-terms approximate solution as

$$
\varphi_{n}=u_{0}+u_{1}+\cdots+u_{n}
$$

with

$$
u(x)=\lim _{n \rightarrow \infty} \varphi_{n}
$$

\section{Conclusion and Discussion}

In this work, we have examined many numerical methods to solve Fredholm integral equations. Using these methods except variational iteration method, the Fredholm integral equations have been reduced to a system of algebraic equations and this system can be easily solved by any usual methods. In this work, we have applied compactly supported semiorthogonal $B$-spline wavelets along with their dual wavelets for solving both linear and nonlinear Fredholm integral equations of second kind. The problem has been reduced to solve a system of algebraic equations. In order to increase the accuracy of the approximate solution, it is necessary to apply higher-order $B$-spline wavelet method. The method of moments based on compactly supported semiorthogonal $B$ spline wavelets via Galerkin method has been used to solve Fredholm integral equation of second kind. This method determines a strong reduction in the computation time and memory requirement in inverting the matrix. Variational iteration method has been successfully applied to find the approximate solution of Fredholm integral equation of both linear and nonlinear types. Taylor series expansion method reduces the system of integral equations to a linear system of ordinary differential equation. After including the required boundary conditions, this system reduces to a system of algebraic equations that can be solved easily. Block-Pulse functions and Haar wavelet method can be applied to the system of Fredholm integral equations by reducing into a system of algebraic equations. These methods give more accuracy if we increase their order. Quadrature method can be applied to solve the nonlinear Fredholm-Hammerstein integral equation of second kind by reducing it to a system of algebraic equations. Homotopy perturbation method (HPM) and Adomian decomposition method (ADM) can be also applied to approximate the solution of nonlinear Fredholm integral equation of second kind. The solutions obtained by HPM and ADM are applicable for not only weakly nonlinear equations, but also strong ones. The approximate solutions by these aforesaid methods highly agree with exact solutions.

\section{References}

[1] A.-M. Wazwaz, Linear and Nonlinear Integral Equations: Methods and Applications, Springer, New York, NY, USA, 2011.

[2] K. Maleknejad and M. N. Sahlan, "The method of moments for solution of second kind Fredholm integral equations based on B-spline wavelets," International Journal of Computer Mathematics, vol. 87, no. 7, pp. 1602-1616, 2010.

[3] J.-H. He, "Variational iteration method-a kind of non-linear analytical technique: some examples," International Journal of Non-Linear Mechanics, vol. 34, no. 4, pp. 699-708, 1999.

[4] J.-H. He, "Some asymptotic methods for strongly nonlinear equations," International Journal of Modern Physics B, vol. 20, no. 10, pp. 1141-1199, 2006.

[5] J.-H. He, "Variational iteration method-some recent results and new interpretations," Journal of Computational and Applied Mathematics, vol. 207, no. 1, pp. 3-17, 2007.

[6] K. Maleknejad, M. Shahrezaee, and H. Khatami, "Numerical solution of integral equations system of the second kind by block-pulse functions," Applied Mathematics and Computation, vol. 166, no. 1, pp. 15-24, 2005.

[7] K. Maleknejad, N. Aghazadeh, and M. Rabbani, "Numerical solution of second kind Fredholm integral equations system by using a Taylor-series expansion method," Applied Mathematics and Computation, vol. 175, no. 2, pp. 1229-1234, 2006.

[8] K. Maleknejad and F. Mirzaee, "Numerical solution of linear Fredholm integral equations system by rationalized Haar functions method," International Journal of Computer Mathematics, vol. 80, no. 11, pp. 1397-1405, 2003.

[9] X.-Y. Lin, J.-S. Leng, and Y.-J. Lu, "A Haar wavelet solution to Fredholm equations," in Proceedings of the International Conference on Computational Intelligence and Software Engineering (CiSE '09), pp. 1-4, Wuhan, China, December 2009.

[10] M. J. Emamzadeh and M. T. Kajani, "Nonlinear Fredholm integral equation of the second kind with quadrature methods," Journal of Mathematical Extension, vol. 4, no. 2, pp. 51-58, 2010.

[11] M. Lakestani, M. Razzaghi, and M. Dehghan, "Solution of nonlinear Fredholm-Hammerstein integral equations by using semiorthogonal spline wavelets," Mathematical Problems in Engineering, vol. 2005, no. 1, pp. 113-121, 2005.

[12] Y. Mahmoudi, "Wavelet Galerkin method for numerical solution of nonlinear integral equation," Applied Mathematics and Computation, vol. 167, no. 2, pp. 1119-1129, 2005.

[13] J. Biazar and H. Ebrahimi, "Iteration method for Fredholm integral equations of second kind," Iranian Journal of Optimization, vol. 1, pp. 13-23, 2009.

[14] D. D. Ganji, G. A. Afrouzi, H. Hosseinzadeh, and R. A. Talarposhti, "Application of homotopy-perturbation method to the second kind of nonlinear integral equations," Physics Letters A, vol. 371, no. 1-2, pp. 20-25, 2007.

[15] M. Javidi and A. Golbabai, "Modified homotopy perturbation method for solving non-linear Fredholm integral equations," Chaos, Solitons and Fractals, vol. 40, no. 3, pp. 1408-1412, 2009. 
[16] S. Abbasbandy, "Numerical solutions of the integral equations: homotopy perturbation method and Adomian's decomposition method," Applied Mathematics and Computation, vol. 173, no. 1, pp. 493-500, 2006.

[17] E. Babolian, J. Biazar, and A. R. Vahidi, “The decomposition method applied to systems of Fredholm integral equations of the second kind," Applied Mathematics and Computation, vol. 148, no. 2, pp. 443-452, 2004.

[18] S. Abbasbandy and E. Shivanian, "A new analytical technique to solve Fredholm's integral equations," Numerical Algorithms, vol. 56, no. 1, pp. 27-43, 2011.

[19] J. C. Goswami, A. K. Chan, and C. K. Chui, "On solving firstkind integral equations using wavelets on a bounded interval," IEEE Transactions on Antennas and Propagation, vol. 43, no. 6, pp. 614-622, 1995.

[20] M. Lakestani, M. Razzaghi, and M. Dehghan, "Semiorthogonal spline wavelets approximation for fredholm integro-differential equations," Mathematical Problems in Engineering, vol. 2006, Article ID 96184, 12 pages, 2006.

[21] C. K. Chui, An Introduction to Wavelets, vol. 1 of Wavelet Analysis and its Applications, Academic Press, Boston, Mass, USA, 1992.

[22] J. C. Goswami and A. K. Chan, Fundamentals of Wavelets, John Wiley \&; Sons, Hoboken, NJ, USA, 2nd edition, 2011.

[23] M. Razzaghi and S. Yousefi, "The Legendre wavelets operational matrix of integration," International Journal of Systems Science, vol. 32, no. 4, pp. 495-502, 2001.

[24] M. Razzaghi and S. Yousefi, "Legendre wavelets direct method for variational problems," Mathematics and Computers in Simulation, vol. 53, no. 3, pp. 185-192, 2000.

[25] A. Ghorbani, "Beyond Adomian polynomials: He polynomials," Chaos, Solitons and Fractals, vol. 39, no. 3, pp. 1486-1492, 2009. 


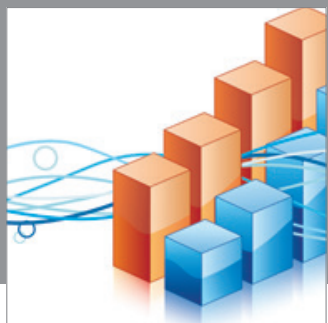

Advances in

Operations Research

mansans

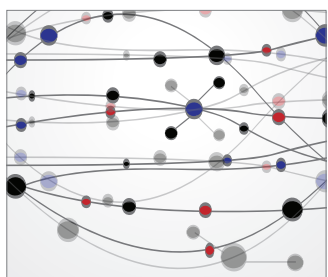

The Scientific World Journal
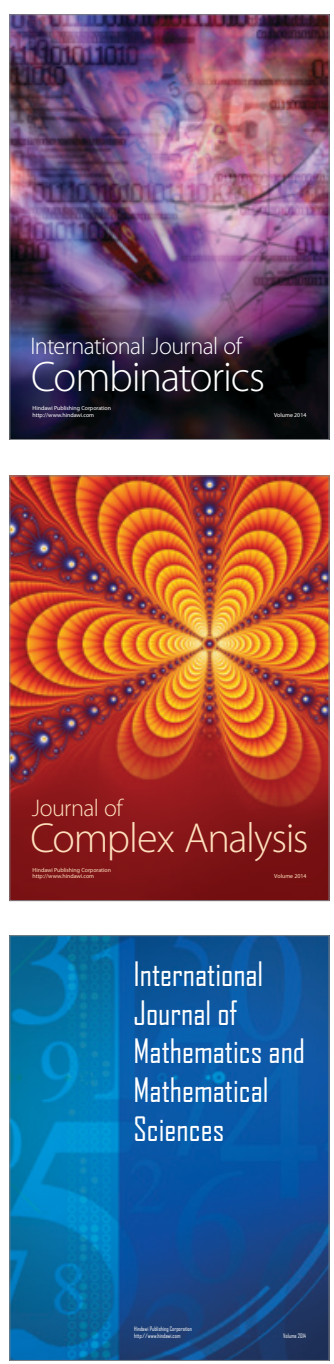
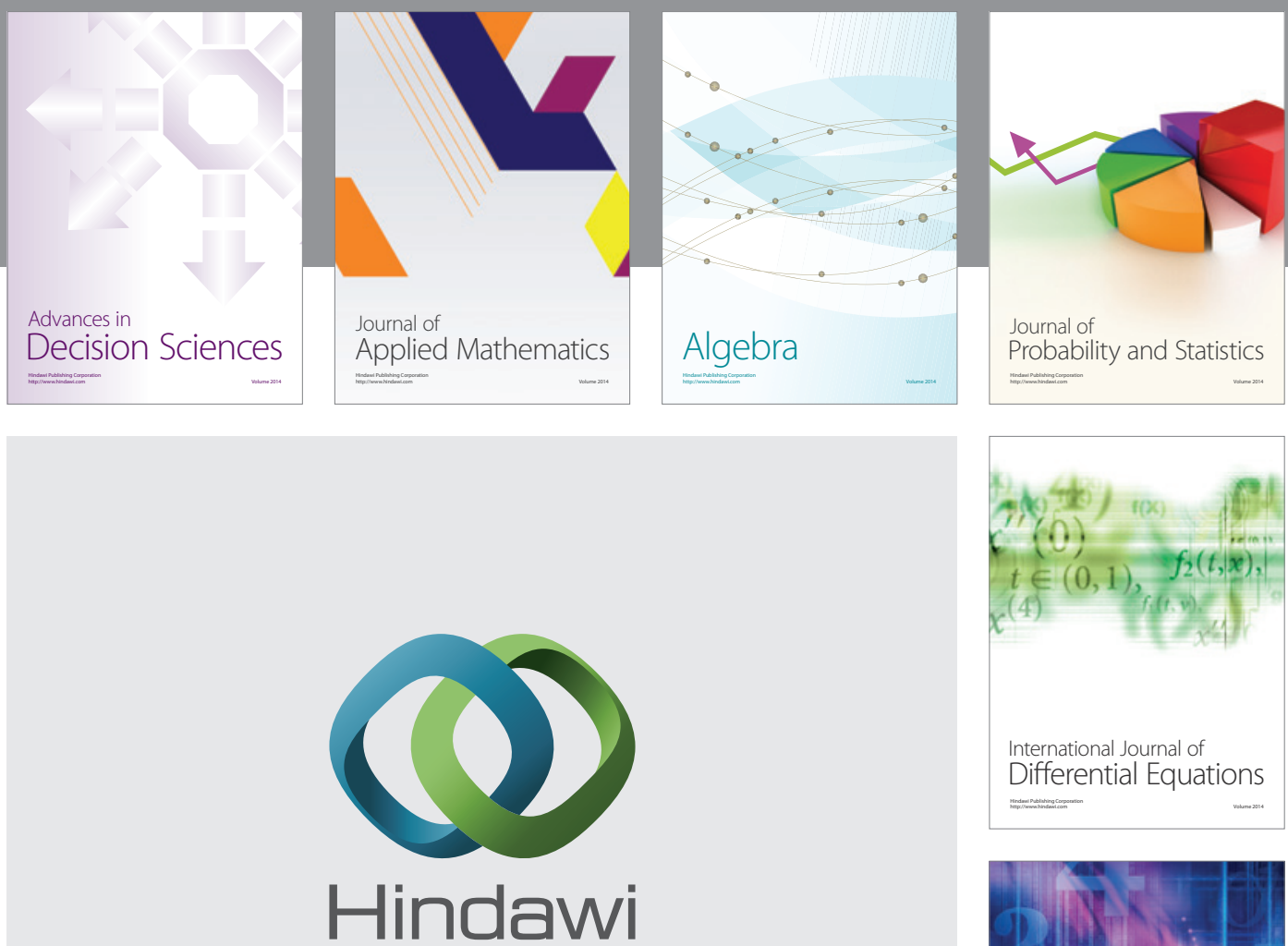

Submit your manuscripts at http://www.hindawi.com
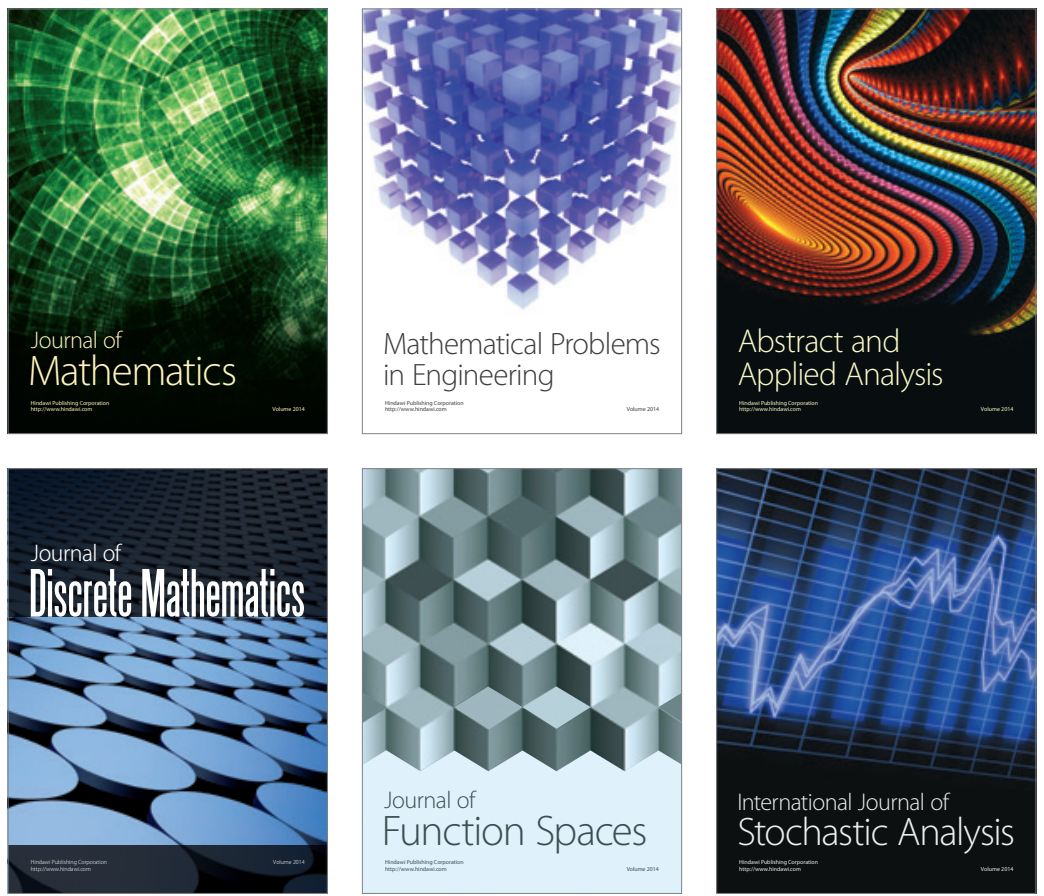

Journal of

Function Spaces

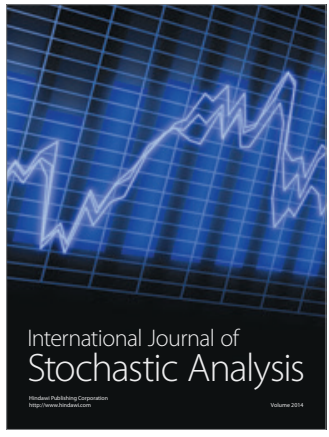

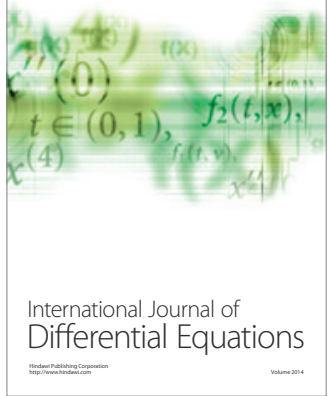
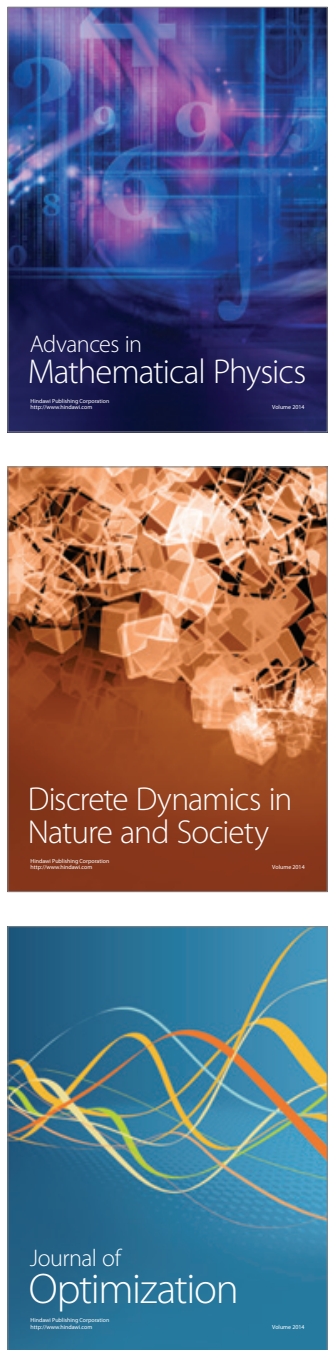